\title{
Responsabilité des professionnels de la santé dans le dépistage de la violence conjugale en temps de pandémie de COVID-19
}

\author{
Nori L. Bradley MD MSc, Ashley M. DiPasquale MD, Kaitlyn Dillabough BSc, Prism S. Schneider MD PhD
}

E Citation : CMAJ 2020 June 1;192:E609-10. doi: 10.1503/cmaj.200634-f; diffusion hâtive le $1^{\text {er }}$ mai 2020

Voir la version anglaise de l'article ici : www.cmaj.ca/lookup/doi/10.1503/cmaj.200634

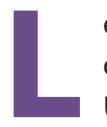

e 27 mars 2020, en raison de la pandémie de maladie à coronavirus 2019 (COVID-19), l'Organisation des Nations Unies a émis une mise en garde contre la hausse des taux de violence conjugale déjà problématiques, y compris des féminicides ${ }^{1}$. Les mesures visant à réduire la propagation du coronavirus du syndrome respiratoire aigu sévère 2 (SRASCoV-2) créent des environnements propices aux comportements qu'un partenaire peut adopter pour exercer un pouvoir et infliger des préjudices physiques, psychologiques ou sexuels à l'autre partenaire ${ }^{2}$. Le stress du confinement, l'incertitude financière, les mentalités concernant les rôles genrés et le désir de contrôle dans un contexte de crise contribuent tous à accroître le risque de violence conjugale ${ }^{3,4}$.

Durant leur récent confinement, la Chine, l'Italie et l'Espagne ont signalé une augmentation substantielle des appels aux services d'écoute téléphonique ${ }^{5}$ pour victimes de violence conjugale. Certains secteurs du Royaume-Uni et de la France ont respectivement constaté des hausses de $20 \%$ et de $30 \%$ du nombre de rapports de police concernant la violence conjugale. Au Canada, les appels aux services d'aide aux femmes battues de la ville de Vancouver ont triplé6, tandis qu'en Alberta, les services d'écoute téléphonique ont enregistré une augmentation de $30 \%$ à $50 \%$ du nombre d'appels. En Ontario, les services de police régionaux de York et de Durham ont quant à eux signalé une augmentation de $22 \%$ des rapports d'incidents familiaux et d'agression sexuelle ${ }^{7}$. Même s'ils ont déjà beaucoup à apprendre en lien avec la COVID-19, les professionnels de la santé doivent aussi rester à l'affût des problèmes de violence conjugale, se tourner vers l'autoformation, développer des stratégies pour aborder la question et se familiariser avec les ressources locales disponibles vers qui orienter leurs patientes.

La violence conjugale est déjà la plus fréquente cause de blessures non fatales infligées aux femmes dans le monde ${ }^{3}$. Même en l'absence d'une crise de santé publique, on estime à $30 \%$ la prévalence de la violence conjugale physique et sexuelle subie par les femmes au cours de leur vie ${ }^{2}$. À l'échelle mondiale,

\section{POINTS CLÉS}

- Les taux de violence conjugale ont augmenté et demeureront un important problème de santé en raison des répercussions économiques de la pandémie et des mesures d'éloignement social requises pour limiter le fardeau de la maladie à coronavirus 2019 pour le système de santé.

- Les professionnels de la santé appelés à soigner des patientes présentant des blessures devraient se familiariser avec les questions de violence conjugale et se renseigner sur les ressources locales disponibles à qui adresser leurs patientes.

- Des campagnes de « mots d'alerte » et d'« Appel à l'aide » ont été lancées pour faciliter les demandes discrètes d'aide dans les cas de violence conjugale compte tenu de l'adoption de plus en plus répandue des consultations par télémédecine.

- Des ressources en ligne validées sont disponibles pour aider les professionnels de la santé à se familiariser et à devenir à l'aise avec le dépistage et le soutien aux patientes victimes de violence conjugale.

tous les 6 jours, une femme est assassinée par son conjoint ${ }^{7}$. Les professionnels de la santé se retrouvent souvent face à des victimes de violence conjugale. Une femme sur 3 qui consulte aux services des urgences pour traumatisme a subi celui-ci aux mains de son partenaire, et 1 femme sur 6 qui consulte pour une fracture a subi de la violence conjugale au cours des 12 mois précédents ${ }^{8}$. Parmi les femmes assassinées par leur partenaire, $45 \%$ avaient consulté un professionnel de la santé pour une blessure infligée par un conjoint violent au cours des 2 années précédant le décès ${ }^{3}$.

Les indicateurs de risque de violence conjugale connus incluent : faible statut socioéconomique, réseau social insuffisant, faible degré d'instruction, toxicomanie, maladie mentale, jeunesse, grossesse non désirée, dépendance financière et statut d'emploi ${ }^{4,9}$. Toutefois, la violence conjugale s'exerce sans égard à la race, à l'origine ethnique, à la classe socioéconomique, à 
l'âge et au type d'union ${ }^{8}$. En raison des préjugés, les personnes victimes de violence conjugale font parfois l'objet de discrimination et sont ainsi laissées à elles-mêmes².

Les traumatismes à la tête et au cou et les blessures aux membres supérieurs sont les lésions physiques plus fréquemment liées à la violence conjugale ${ }^{3}$. Malheureusement, seulement $14 \%$ des patientes qui consultent pour des blessures liées à la violence conjugale se font interroger sur l'origine du problème et proposer du soutien ${ }^{9}$. Pour les patientes que le professionnel de la santé soupçonne d'être victime de violence conjugale, les données probantes préconisent des questions directes, en toute discrétion ${ }^{9}$. La plupart des femmes ne livrent pas spontanément ce type d'information'. Il revient aux professionnels de la santé d'engager le dialogue avec elles. La position de l'American College of Surgeons au sujet de la violence conjugale enjoint les chirurgiens à " non seulement soigner les blessures des patientes quand elles consultent, mais également à les orienter vers les ressources appropriées et à faire un suivi $»^{10}$. Les associations canadiennes et américaines d'orthopédie encouragent aussi leurs membres à se former sur le sujet afin de pouvoir dépister la violence conjugale et aider leurs patientes ${ }^{11}$.

Il existe des outils et des ressources validés pour aider les médecins à dépister les nouveaux cas de violence conjugale et pour soutenir les pratiques et les programmes là où ils sont défaillants. Le programme EDUCATE (www.ipveducate.com/ the-educate-training-program) est une plateforme de formation multimodale qui propose des ressources aux chirurgiens orthopédistes et autres professionnels œuvrant auprès de patientes victimes de traumatismes afin qu'ils se familiarisent et deviennent plus à l'aise avec le dépistage et le soutien aux patientes ${ }^{8}$. L'initiative albertaine REAL Talk (https://realtalk. sagesse.org/) offre aux professionnels de la santé et au public une formation et des ateliers en ligne axés sur la sensibilisation, l'empathie et l'écoute active des personnes touchées. Pour sa part, l'Hôpital Women's College de Toronto propose un programme de formation sur la violence conjugale (http:// dveducation.ca), incluant des modules d'autoformation en ligne pour acquérir les compétences cliniques relatives aux différents aspects de la violence conjugale. Dans son énoncé de position sur la violence conjugale, l'American College of Surgeons préconise la technique SAFE (Sécurité/stress, Abus/ peur, Famille/amis, en urgence) (www.facs.org/about-acs/ statements/115-partner-violence) ${ }^{10}$.

L'incertitude quant à la façon de gérer la divulgation d'une situation de violence conjugale est un obstacle fréquent à son dépistage ${ }^{11}$. Les plateformes éducatives, comme EDUCATE, peuvent aider le médecin à se sentir prêt et à l'aise de composer avec une situation de divulgation ${ }^{8}$. Le programme REAL Talk, le manuel clinique de l'Organisation mondiale de la Santé ${ }^{12}$ et le programme EDUCATE offrent tous de la formation sur la façon de veiller à la sécurité de la patiente dans l'immédiat, y compris la mise en place d'un plan de sécurité, l'orientation vers les services sociaux et d'autres ressources appropriées.

En raison de la pandémie de COVID-19, il est plus difficile dans un contexte ambulatoire d'identifier la violence conjugale et d'offrir de l'aide. De nombreux médecins et chirurgiens optent maintenant pour les suivis en télémédecine afin de maintenir l'éloignement physique. Or, les suivis par téléphone ou vidéoconférence avec les patientes à la maison se font souvent alors qu'elles sont confinées avec leur agresseur. Dans la foulée d'une initiative européenne pour aider les patientes qui ne peuvent pas être interrogées privément à informer leurs professionnels de la santé si elles sont à risque, une campagne canadienne a été lancée autour du « mot d'alerte ${ }^{13}$. La campagne "Appel à l'aide » prévoit un geste de la main pour demander silencieusement de l'aide lors d'une consultation de télémédecine (https:// canadianwomen.org/fr/appel-a-l-aide/). L'objectif est d'offrir des stratégies de communication discrètes pour les patientes qui souhaitent obtenir de l'aide. Si une patiente utilise le mot d'alerte ou le geste d'appel à l'aide, le professionnel de la santé devrait lui offrir son soutien et l'orienter vers les services locaux d'aide aux femmes victimes de violence conjugale. La campagne «Appel à l'aide » offre des trousses et des stratégies pour la consultation sécuritaire de son site Web.

Les Nations Unies ont enjoint les gouvernements à poursuivre leur lutte contre la violence conjugale en temps de COVID-19, afin que soit maintenu l'accès à des services juridiques, à des refuges sécuritaires et à des services d'écoute téléphonique pour les personnes touchées ${ }^{1}$. Le gouvernement fédéral canadien a annoncé un financement supplémentaire de 40 millions de dollars versé aux refuges pour femmes et aux centres pour victimes d'agression sexuelle, et de 10 millions de dollars pour les femmes et les enfants autochtones qui fuient la violence ${ }^{14}$. Donc, les refuges pour femmes au Canada demeureront ouverts, et disposeront de programmes de dépistage de la COVID-19 sur place. Toutefois, les stratégies de dépistage et d'aide doivent aussi inclure d'autres options, telles que le clavardage et les textos pour une communication sécuritaire en temps de confinement. Une fois la pandémie passée, la crise financière perdurera pendant des mois et exacerbera le risque de violence conjugale. Durant les 9 mois qui ont suivi le passage de l'ouragan Katrina, le taux de violence faite aux femmes par des partenaires a été 3 fois plus élevé dans la région touchée que le taux annuel dans le reste des États-Unis, et les viols commis par les conjoints ont été multipliés par $16^{4}$.

Tous les professionnels de la santé doivent se préparer à une augmentation de la violence conjugale associée à la pandémie de COVID-19 et à ses séquelles.

\section{Références}

1. States must combat domestic violence in the context of COVID-19 lockdowns - UN rights expert [press release]. Geneva: Office of the United Nations High Commissioner for Human Rights; 2020 Mar. 27. Accessible ici : www.ohchr.org/ EN/NewsEvents/Pages/DisplayNews.aspx?NewsID=25749\&LangLa=E (consulté le $1^{\text {er }}$ avril 2020).

2. Garcia-Moreno C, Hansen HAFM, Ellsberg M, et al. Prevalence of intimate partner violence: findings from the WHO multi-country study on women's health and domestic violence. Lancet 2006;368:1260-9.

3. Bhandari M, Dosanjh S, Tornetta P III, et al.; Violence Against Women Health Research Collaborative. Musculoskeletal manifestations of physical abuse after intimate partner violence. J Trauma 2006;61:1473-9.

4. World disasters report 2007 - focus on discrimination. Geneva: International Federation of Red Cross; 2007. Accessible ici : www.ifrc.org/en/publicationsand-reports/world-disasters-report/wdr2007/ (consulté le $1^{\text {er }}$ avril 2020). 
5. Taub A. A new COVID-19 crisis: domestic abuse rises worldwide. The New York Times le 6 avril 2020. Accessible ici : www.nytimes.com/2020/04/06/world/ coronavirus-domestic-violence.html (consulté le 22 avril 2020).

6. Daya R, Azpirir J. Calls to Vancouver domestic-violence crisis line spike $300 \%$ amid COVID-19 pandemic. Global News le 7 avril 2020. 7. Accessible ici : https://globalnews.ca/news/6789403/domestic-violence-coronavirus/ (consulté le 7 avril 2020).

7. Amin F. Domestic violence calls surge during pandemic. City News [Toronto] le 8 avril 2020. Accessible ici : https://toronto.citynews.ca/2020/04/08/domestic -violence-calls-surge-during-coronavirus-pandemic/ (consulté le 22 avril 2020).

8. EDUCATE Investigators. Novel education program improves readiness to manage intimate partner violence in the fracture clinic: a pretest-posttest study. CMAJ Open 2018;6:E628-36.

9. PRAISE Investigators; Sprague S, Bhandari M, Della Rocca GJ, et al. Prevalence of abuse and intimate partner violence surgical evaluation (PRAISE) in orthopaedic fracture clinics: a multinational prevalence study. Lancet 2013;382:866-76.

10. Statement on intimate partner violence. Chicago: American College of Surgeons; mise-à-jour le $1^{\text {er }}$ juin 2018. Accessible ici : www.facs.org/about-acs/ statements/115-partner-violence (consulté le $1^{\text {er }}$ avril 2020).

11. Della Rocca GJ, Tornetta P III, Schneider PS, et al. Intimate partner violence and orthopaedics: AOA critical issues. J Bone Joint Surg Am 2019;101:e62.

12. Health care for women subjected to intimate partner violence or sexual violence: a clinical handbook. Geneva: World Health Organization. Accessible ici : https://apps.who.int/iris/bitstream/handle/10665/136101/WHO_RHR_14.26_ eng.pdf? sequence=1 (consulté le 22 avril 2020).

13. Higgins N. Coronavirus: when home gets violent under lockdown in Europe. $B B C$ News [Brussels] le 13 avril 2020. Accessible ici : www.bbc.com/news/world -europe-52216966 (consulté le 27 avril 2020).

14. Canada announces support to those experiencing homelessness and women fleeing gender-based violence during the coronavirus disease (COVID-19) pandemic [news release]. Le 4 avril 2020. Gatineau (QC): Employment and Social Development Canada. Accessible ici : www.canada.ca/en/employment-social-development/news/ 2020/04/canada-announces-support-to-those-experiencing-homelessness-and -women-fleeing-gender-based-violence-during-the-coronavirus-disease-covid-19 -pandemic.html (consulté le 4 avril 2020).

\section{Intérêts concurrents : Aucun déclaré.}

Cet article a été révisé par des pairs.

Affiliations : Département de chirurgie (Bradley, DiPasquale), Université de l'Alberta, Edmonton, Alta.; Département de médecine de soins intensifs (Bradley), Université de la Colombie-Britannique, Vancouver, C.-B.; Département de chirurgie (Dillabough) et Départements de chirurgie et de médecine communautaire (Schneider), Faculté de médecine Cumming de l'Université de Calgary, Calgary, Alta.

Collaborateurs : Tous les auteurs ont contribué à la conception du travail, ont rédigé le manuscrit, en ont révisé de façon critique le contenu intellectuel important; ils ont donné leur approbation finale pour la version destinée à être publiée et assument l'entière responsabilité de tous les aspects du travail.

Correspondance : Prism Schneider, prism.schneider@ albertahealthservices.ca 